\title{
Qualitätsanforderungen bei der Laserbearbeitung metallischer Präzisionsfolien
}

\author{
Asta Richter, Dobri Erinski
}

\begin{abstract}
Metallpräzisionsfolien finden eine immer breitere Anwendung in vielen Bereichen der modernen Fertigung. Materialstärken von $5 \mu \mathrm{m}$ bis $300 \mu \mathrm{m}$ sind für ein breites Spektrum von Anwendungen interessant. Dabei werden hohe Qualitätsanforderungen gestellt: Maßtoleranzen im Bereich von wenigen Mikrometern, gratarme Konturen und beeinträchtigungsfreie Funktionsoberflächen. Die mehrlagige Bearbeitung flacher Werkstuicke bringt beachtliche Vorteile, u. a. gratarme Schnittkanten höchster Qualität. In diesem Fall werden mehrlagige Folienpakete gleichzeitig aufgespannt und gelasert. Die gezielte Anwendung von Haltefolien stabilisiert das Folienpaket im Gasstrom und erhöht somit die Fertigungsqualität. Um die Trennung der Folienlagen zu ermöglichen und um die angestrebte hohe Oberflächenqualität zu erreichen, sind geeignete Trennmittel zu verwenden.
\end{abstract}

Metal precision foils have a wide variety of applications in the recent fields of production technology. The foils considered here have a thickness between $5 \mu \mathrm{m}$ and 300 $\mu \mathrm{m}$. Quality features of the laser cutting contours are measuring tolerances within a few micrometers, cutting contour edges with a minimum burr, and proper function surfaces without defects. Multilayer laser processing of flat workpieces is economic, of high efficiency and has the advantage of laser cutting edges with high quality. Stacks of foils are put together and machined by the laser. The straightforward application of support foils stabilises the foil pile within the gas flow and increases the laser machining quality. The separation of the single precision foils of the stack is attained through use of special materials such as supporting separation lubricant fluids.

\section{Einführung in die Problematik}

Metallpräzisionsfolien mit Materialstärken im Bereich von $5 \mu \mathrm{m}$ bis $300 \mu \mathrm{m}$ finden eine immer breitere Anwendung in vielen Bereichen der modernen Fertigungstechnik. Für das Bearbeitungsergebnis beim Laserschneiden, eine inzwischen auf vielen Gebieten etablierte Technologie mit immer noch erheblichem Entwicklungspotenzial, ist das Zusammenwirken von Laserlicht und Prozessgas von entscheidender Bedeutung. Die mechanische Belastung der Metallfolien durch das mit einem definierten Druck ausströmende Prozessgas bestimmt in entscheidendem Maße die Qualität des Laserschneidens, da dadurch lokal die Lage der Laserstrahl-Fokusebene signifikant beeinflusst wird $[1,3,6]$.
Für jeden konkreten Einsatzfall ergeben sich spezifische Anforderungen sowohl an die Materialeigenschaften als auch an die Fertigungsgenauigkeit und die Oberflächengiite. Darüber hinaus gilt, dass die Außen- wie Innenkonturen einschließlich Öffnungen und Einschnitte mit den vorgegebenen, meist engen Maßtoleranzen hergestellt werden miissen.

Die Fertigungsqualität der Metallpräzisionsfolien wird durch die Ausbildung der Schnittkanten entscheidend beeinflusst, da hier die Wechselwirkung Schneidwerkzeug - Werkstoff zu lokalen Veränderungen der Werkstoffeigenschaften führen kann. Gratbildung und plastische Verformung sowie erhöhte Rauheit verschlechtern die Gebrauchseigenschaften. Störend können sich auch Gefügeveränderungen im Schnittkantenbereich auswirken. Lokale Veränderungen der chemischen Eigenschaften können z. B. eine Korrosion des Folienmaterials bewirken. Aus diesen Gründen ist es in jedem Fall notwendig, bei der Herstellung von Präzisionsfolien den Schnittkantenbereich umfassend zu charakterisieren und die eingesetzte Laserschneidtechnologie qualitätsbezogen zu optimieren [10 - 12].

\section{Laseranlage, Bearbeitungsparameter, Handhabung der Folienwerkstücke}

Die Untersuchungen zum Laserschneiden dünner Metallpräzisionsfolien wurden an einer LaserWorkStation LWS 440 mit einem gepulsten Nd:YAG-Festkörperlaser vorgenommen. Es wurde mit der Standardwellenlänge des Lasers bei $1064 \mathrm{~nm}$ gearbeitet. Die Pulsdauer konnte zwischen 0,1 und $20 \mathrm{~ms}$ bei Pulsfrequenzen von 0,1 bis $1000 \mathrm{~Hz}$ variiert werden. Die Pulsenergie betrug max. $30 \mathrm{~J}$, so dass dann die mittlere Laserleistung bei $150 \mathrm{~W}$ lag. Die resultierenden Pulsenergiewerte beim Lasern von Metallpräzisionsfolien lagen im Bereich unterhalb von $0,1 \mathrm{~J}$, für Folienstärken von 5 bis $50 \mu \mathrm{m}$ sogar unterhalb von $0,05 \mathrm{~J}$.

Metallfolien im oberen untersuchten Materialstärkenbereich ( $\mathrm{um} 300 \mu \mathrm{m}$ ) bereiten weder bei der Handhabung noch bei der Aufspannung besonders große Schwierigkeiten. Sie lassen sich mit den bekannten konventionellen Aufspann-Techniken plan sowie stabil befestigen und während des Laserschneidens in dieser Lage festhalten. Ein Defokussieren aus diesem Grund ist bei den genannten Folienstärken so gut wie ausgeschlossen.

Bei Folien im unteren untersuchten Materialstärkenbereich treten mit Abstand die größten Probleme gera- 
de bei der Handhabung und der Aufspannung der Werkstiicke auf. Liegt die Laserstrahl-Fokusebene auf der Oberfläche $(\mathrm{AF}=0)$ oder darunter (Minuslage der Fokusebene), so kann das Laserschneiden, keine sonstigen negativen Einflüsse vorausgesetzt, überaus erfolgreich sein. Liegt jedoch die Fokusebene darüber und ist die Tiefenschärfe des Laserstrahls unter den gewählten Bearbeitungsbedingungen relativ gering, so vermindert sich entweder die Laserschneidqualität gravierend oder aber der Vorgang des Laserschneidens selbst wird lokal unterbrochen $[1,7,8]$.

Weitere negativ beeinflussende Faktoren sind die thermische Ausdehnung der metallischen Teile des Arbeitstisches sowie der Fixier- und Aufspann-Vorrichtung, Maßabweichungen oder Unebenheiten bei der Befestigung dieser Vorrichtung, ferner die Kräfte, die infolge des Schmelzens bzw. des Sublimierens des Schnittspaltmaterials während des Laserschneidvorgangs entstehen und auf das Folienwerkstiick einwirken.

Beim Laserschneiden werden in der Regel als Prozessgase Sauerstoff (Brennschneiden) oder Inertgase (Schmelzschneiden, Sublimierschneiden) eingesetzt [13]. Das Prozessgas strömt koaxial zum fokusierten Laserstrahl unter einem definierten Druck auf das zu bearbeitende Material. Dadurch kann dieses lokal aus der Fokusebene des Laserstrahls gedrückt werden. Eine FokusNachfuihrung mit Hilfe von Abstandssensoren ist bei dünnen Metallfolien wenig sinnvoll, da hier ein über die Sensorfläche integriertes Signal lediglich eine flächengemittelte, ungenaue wie auch ziemlich träge Abstandskorrektur gestattet.

Die vertikale Bewegung (Flattern) des Folienmaterials während des Schneidvorgangs, die hauptsächlich durch den zum Bearbeiten erforderlichen Gasstrom verursacht wird, stellt das Hauptproblem beim Laserschneiden von Metallfolien im unteren Dickenbereich dar. Dadurch unterliegen die Leistungsdichte des Laserstrahls und der Schneidgasstrom selbst hohen Schwankungen, was sich negativ auf die Schneidkantenqualität auswirkt und zur erhöhten Grat- oder Lotperlenbildung sowie zu beachtlichen Konturungenauigkeiten führt $[1,6]$. Bei erheblichem Defokussieren kann außerdem eine Unterbrechung des Schneidvorgangs stellenweise oder ganz eintreten, was ebenso zu irreparablen Schäden fuihrt.

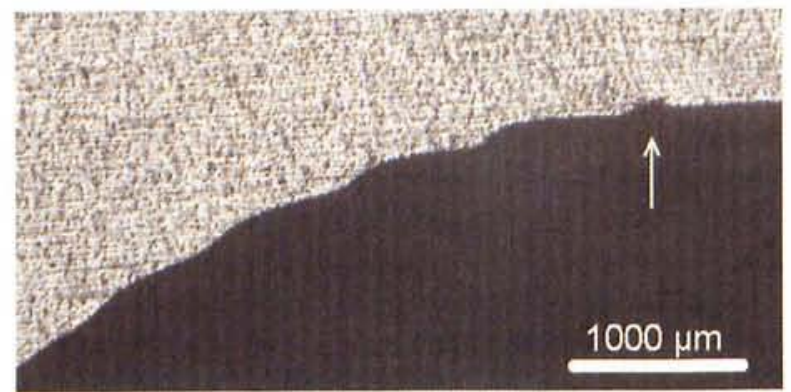

Abb. I: Korrosionsbeständiger, austenitischer Stahl X10CrNi18-8 mit einer Materialstärke von $10 \mu \mathrm{m}$; Pulsfrequenz $650 \mathrm{~Hz}$; Spannung $205 \mathrm{~V}$; Pulsdauer $102 \mu \mathrm{s}$; Fokusabstand 1,0 mm; Fokuslage - $5 \mu \mathrm{m}$; Prozessgas Stickstoff; Druck 2 bar; Düsendurchmesser $1,0 \mathrm{~mm}$; Schneidgeschwindigkeit $1.500 \mathrm{~mm} / \mathrm{min}$; Trockenschnitt.
Das Phänomen des „Flatterns“ tritt besonders störend beim Laserschneiden innenliegender, geschlossener Konturen auf, wie bei Bohrungen oder Ausschnitten (Bild 1), da diese von unten meist nicht gestiutzt werden können. Der Laserstrahl-Einstichkrater mit nachfolgendem geradem Laserschnitt im Uhrzeigersinn (rechts oben) sowie die ausgeprägte wellenförmige Kontur, die durch das gasdruckbedingte Flattern der Folie kurz vor dem Bearbeitungsende und durch die dabei stattfindende permanente Abbremsung der Vorschubgeschwindigkeit entstand (linker Teil), sind in Abbildung 1 deutlich erkennbar.

Der Einsatz von speziellen, für jede Probenform anders geformten Schneidunterlagen und das Auflegen von Folienbefestigungen kann das Flattern erheblich mindern, nicht jedoch vollständig unterdrücken. Für Metallfolien im unteren untersuchten Dickenbereich muss daher eine problemangepasste Halterungstechnik eingesetzt werden [6]:

- mechanische, magnetische oder pneumatische Fixierund Aufspann-Vorrichtungen für die zu schneidenden Metallpräzisionsfolien,

- außenkonturangepasste Unterlagen aus unterschiedlichen Materialien,

- außen- wie innenkonturangepasste Unterlagen mit Befestigungsbrïcken für die freiliegenden Unterlagen-Elemente,

- zwei- bzw. mehrgeteilte Fixier- und Aufspann-Vorrichtungen, bestehend aus Oberteil (angepasst an die Außenkonturen) und Unterteil (Unterlagen, die an allen Außen- wie Innenkonturen angepasst sind sowie aus einer Lage oder aus mehreren Blechtafeln bestehen können),

- Befestigungselemente in Form von konturangepassten Auflagen.

\section{Laserschneiden von Metallpräzisions- folien im Trockenschnitt}

Beim Laserschneiden von Metallpräzisionsfolien im Trockenschnitt wird die erreichbare Schnittflächenguite hauptsächlich durch die Gratbildung an der Unterkante der Schnittkonturen bestimmt. Die Metallpräzisionsfolien werden unter Anwendung von Inertgasen wie Stickstoff, Argon oder Helium gelasert. Das Bearbeitungsverfahren wird in diesem Fall als Schmelzschneiden bezeichnet. Die während des Laserschneidens gebildeten Gratanhaftungen bestehen aus dem Metall, das geschnitten wird. Der Grat entlang der Unterseite der Schnittkontur entsteht durch einen unzureichenden Schmelzaustrieb an der Schneidfront. Dem kann durch die folgenden Maßnahmen erfolgreich begegnet werden [1]:

1. Steigerung des Schneidgasdruckes, z. B. bei Stickstoff bis zu 20 bar (HD-Schneiden),

2. Vergrößerung der Schnittspaltbreite, z. B. durch Vergößerung des Fokusdurchmessers,

3. Verlegung der Lage der Fokusebene von der Strahleintrittsoberfläche (Standardlage) in Richtung zur Strahlaustrittsoberfläche der Folie (Minuslage der Fokusebene), wodurch sich die Schnittspaltbreite deutlich vergrößert, 
4. Änderung der parallelen Spaltform zu einer leicht konischen, sich nach unten verjüngenden Form des Schnittspaltes,

5. Steigerung der Leistungsdichte im unteren Schnittspaltbereich,

6. Verringerung der Pulsdauer, dadurch Verminderung der Pulsenergie,

7. Erhöhung der Schneidgeschwindigkeit bis in den Bereich der maximal möglichen Geschwindigkeiten, bei welchen der Vorgang des Laserschneidens noch stattfinden kann,

8. Anwendung von Schutzfolien, d. h. Unterlegung der Werkstïckfolie mit einer „verlorengehender“ Hilfsfolie, die mit durchgeschnitten wird und an der der Grat haftet. Die Schutzfolie wird nach dem Laserschneiden vom Werkstïck entfernt $[1,3,6]$.

Die aufgeführten Maßnahmen zur Verringerung der Gratbildung und somit zur Steigerung der Fertigungsqualität (bis auf die Anwendung von Schutzfolien) dienen einem effizienteren Schmelzaustrieb und/oder verringern die Zähigkeit der Schmelze im unteren Schnittflächenbereich an der Schneidfront.

Es ist zu beachten, dass sowohl die einzelnen Maßnahmen wie auch die beliebigen Maßnahmen-Kombinationen bestenfalls eine erhebliche Verminderung, nicht jedoch die vollständige bzw. die weitestgehende Unterdruickung der Gratbildung beim Laserschneiden von Metallpräzisionsfolien bewirken können. Ein beachtlicher Qualitätssprung im Hinblick auf die Unterdrückung der Gratbildung läßt sich nur durch Einsatz von Trennmitteln während des einlagigen bzw. mehrlagigen Laserschneidens realisieren $[1-6,9]$.

Die geforderte hohe Fertigungsqualität beim Laserschneiden von Metallpräzisionsfolien aus Nickelbasislegierungen (z. B. NiCr15Fe, d. h. Inconel 600 mit einer Materialstärke von $127 \mu \mathrm{m}$ bzw. $200 \mu \mathrm{m}$ ) bzw. hochschmelzenden Metallen (z. B. ReinWolfram mit einer Materialstärke von $100 \mu \mathrm{m})$ läßt sich nur im Trockenschnitt erreichen, da durch den Einsatz von Trennmitteln eine sprunghafte Abkiihlung der Strahlaustrittsoberfläche verursacht wird, die wiederum die Leistungsdichte in diesem Bereich erheblich senkt. In diesem Fall nimmt die Intensität der Gratbildung stark zu, da infolge der Abkuihlung die Zähigkeit der Schmelze im unteren Schnittflächenbereich an der Schneidfront beträchtlich zunimmt [7].

Durch die Anwendung der genannten gratbildunghemmenden Maßnahmen ( 1 bis 7) lassen sich beim Laserschneiden von Metallpräzisionsfolien im Trockenschnitt sehr gute Ergebnisse erzielen. Als Beispiel sei das Hochdruck-Laserfeinschneiden von kreisförmigen Rohlingen aus der Nickelbasislegierung Inconel 600 angefuihrt (Abbildung 2).

Bei diesen Untersuchungen galt es, kreisförmige Rohlinge mit einem Durchmesser von $(170 \pm 5) \mu \mathrm{m}$ aus NiCr15Fe-Folien mit einer Dicke von $127 \mu \mathrm{m}$ herzustellen. Die außerordentlich schmale Schnittspaltbreite beträgt nur $28 \mu \mathrm{m}$, der rechnerisch ermittelte

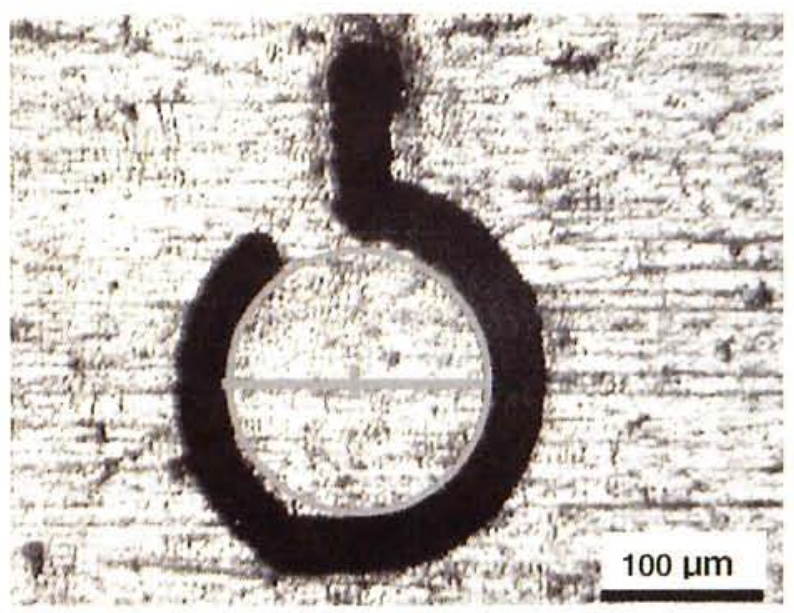

Abb. 2: Nickelbasislegierung NiCr15Fe (Inconel 600) mit einer Materialstärke von $127 \mu \mathrm{m}$; Pulsfrequenz $500 \mathrm{~Hz}$; Spanmung $230 \mathrm{~V}$; Pulsdauer $103 \mu \mathrm{s}$; Fokusabstand $0.6 \mathrm{~mm}$, Fokuslage-20 $4 \mathrm{~m}$; Prozessgas Stickstoff; Druck $19 \mathrm{bar}$; Diësendurchmesser $0.5 \mathrm{~mm}$; Schneidgeschwindigkeit $6 \mathrm{~mm} / \mathrm{min}$; Trockenschnitt. Die Rohlinge werden mit einem Verbindungssteg gelasert und erst beim Einsatz aus der Folie herausgetrennt.

Durchmesser-Mittelwert liegt mit $166 \mu \mathrm{m}$ innerhalb der geforderten Maßtoleranzen.

Das Hochdruck-Laserfeinschneiden von ReinWolframFolien mit einer Materialstärke von $100 \mu \mathrm{m}$ im Trockenschnitt erwies sich als ein überaus erfolgversprechendes Laserbearbeitungsverfahren, um auf diesem Wege den gestellten sehr hohen Qualitätsanforderungen hinsichtlich Maßtoleranzen und Oberflächengüte gerecht zu werden (Abbildung 3).
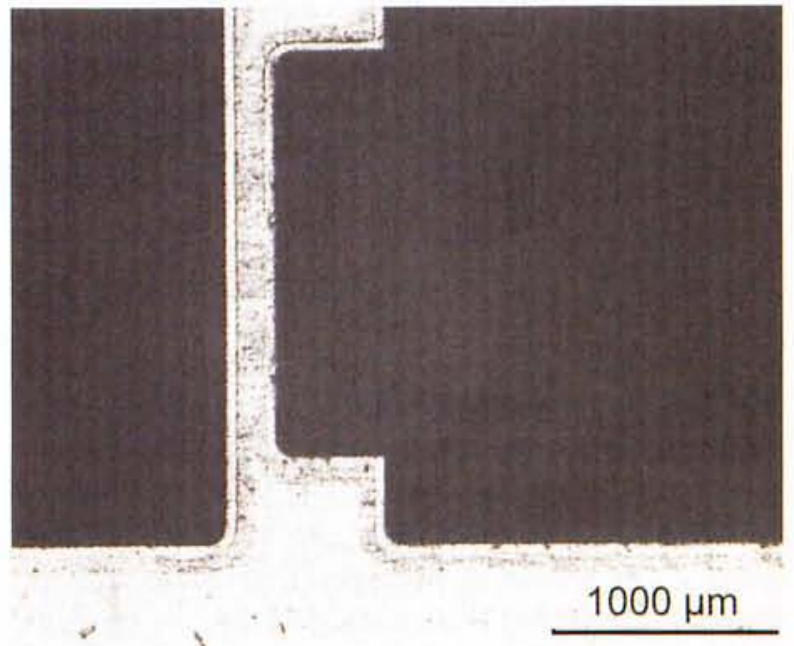

Abb. 3: Mikrokonturen einer Bedampfungsmaske aus ReinWolfram-Folie (99,96: W) mit einer Materialstärke von $100 \mu \mathrm{m}$; Pulsfrequenz $120 \mathrm{~Hz}$; Spannung $300 \mathrm{~V}$; Pulsdauer $250 \mu \mathrm{s}$; Fokusabstand 0.2 mm; Fokuslage -35 $\mu \mathrm{m}$ : Prozessgas Stickstoff: Druck 18 bar; Düsendurchmesser $0.5 \mathrm{~mm}$; Schneidgeschwindigkeit $16 \mathrm{~mm} / \mathrm{min}$; Verfahren Hochdruck-Laserfeinschneiden im Trockenschnitt; Stegbreite (Bildmitte) Sollwert 220-0/ + $20 \mu$ m; absolute Toleranzbreite DT $=20 \mu \mathrm{m}$; Istwert $225 \mu \mathrm{m}$.

Das verwendete Folienmaterial aus ReinWolfram $(99,96 \%$ W) wurde pulvermetallurgisch hergestellt; anschließend wurden die Wolfram-Folienbänder in mehreren Umformvorgängen erzeugt. Die letzten Stufen der plastischen Verformung fanden nachweislich unterhalb der Rekristallisationstemperatur des metallischen Werkstoffs statt und verliefen nicht unter den Bedingungen einer Schutzgas-Atmosphäre. 
Das besagte ReinWolfram-Folienmaterial wurde nach Beendigung der Umformvorgänge nicht oder nur unvollständig einem sonst üblichen Glühvorgang (z. B. Rekristallisationsgliihen, Spannungsfreigliihen, Erholungsgliihen) unterworfen. Demzufolge waren die mechanischen Eigenschaften des verwendeten Folienmaterials ausgesprochen heterogen. Die ReinWolfram-Folie wies einen auch im Makrobereich feststellbaren, grobkörnigen Gefügeaufbau und zahlreiche lokale OberflächenUnebenheiten auf [7].

Das Wolfram-Folienmaterial war ausgesprochen spröde, wies eine relativ hohe Mikrohärte auf und ließ sich leicht durch Biegen zum Bruch bringen. Die erheblichen Restspannungen im Wolfram-Folienmaterial waren ausgesprochen ungleichmäßig verteilt.

Aus praktischen Erfahrungen mit durch plastische Verformung erzeugten Folienbändern ist bekannt, dass die Restspannungen in rekristallisationsgeglïhten Bändern relativ gering sind, keine bevorzugte Richtungsabhängigkeit aufweisen und keine nennenswerten Verformungen in Z-Richtung (DZ ist dann entweder vernachlässigbar gering oder aber gleich Null) bei den nachfolgenden Bearbeitungsstufen verursachen. Der mathematische Ort für Restspannungen in der XY-Ebene rekristallisationsgeglïhter Folien ist durch einen Kreis definiert. Aus diesem Grund verbleiben bei derartigen Metallfolien die Abmessungen vor, während und nach dem Laserschneiden in jeder Richtung nahezu unverändert [7].

Im gelaserten ReinWolfram-Folienmaterial waren dagegen die Restspannungen beachtlich. Zudem wiesen sie eine bevorzugte Richtungsabhängigkeit auf und verursachten bei den nachfolgenden Bearbeitungsstufen erhebliche, zum Teil untragbar hohe Verformungen in ZRichtung (die DZ-Werte waren nicht gleich Null und lagen sogar deutlich über den tolerierbaren Abweichungswerten für vergleichbare Metallfolien). Der mathematische Ort für Restspannungen in der XY-Ebene derartiger Folienwerkstüicke ist durch eine Ellipse definiert - anders in der Walzrichtung im Vergleich zur Querrichtung. Nach dem Fertiglasern resultieren dadurch messbare Abmessungsveränderungen in allen Richtungen. Die häufigste Erscheinungsform derartiger Restspannungszustände ist die sogenannte "sattelförmige" Verformung flacher Werkstiickbereiche, die bei allen fertiggelaserten Bedampfungsmasken beobachtet wurde [7].

Die erhöhte Mikrohärte in bestimmten Folienbereichen wie auch die erhebliche Bruchneigung des gesamten Folienmaterials führten außerdem dazu, dass während des Laserns Überbeanspruchungen der diinnsten Stegbereiche in den gelaserten Maskenwerkstiicken stattfanden. So waren in manchen Werkstïcken mehrere angerissene, durchgebrochene bzw. gar mit ausgebrochenen Fehlstellen versehene Stege mit der Soll-Abmessung (220 \pm 10$) \mu \mathrm{m}$ vorhanden. Aus diesem Grund dürfte davon ausgegangen werden, dass diese dünnen Stege außerordentlich unstabil, stoßempfindlich und bruchanfällig sind.
Das Einhalten der vorgegebenen engen Maßtoleranzen (bei vielen Abmessungen $\pm 25 \mu \mathrm{m}$ oder absolut DT $=$ $50 \mu \mathrm{m}$, bei anderen Abmessungen $\pm 15 \mu \mathrm{m}$ oder absolut DT $=30 \mu \mathrm{m}$ bzw. betreffs des Dünnstegs von $220 \mu \mathrm{m}$ lediglich $-0 \mu \mathrm{m} /+20 \mu \mathrm{m}$ oder eine absolute Toleranzbreite DT $=20 \mu \mathrm{m}$ ) war außerordentlich schwierig, vor allem deshalb, weil sich die als plan angenommenen Folienbereiche während des Laserschneidens in Z-Richtung unvorhersehbar wie auch unzulässig stark verformt oder aber hin und her bewegt haben [7].

Bei einem bestimmten, durch die festgelegten, vorher optimierten Laserstrahl-Formungsparameter beeinflussten Laserstrahldurchmesser können die zu erzeugenden Konturen ziemlich genau vorhergesagt, berechnet und auch gelasert werden. Unabdingbare Voraussetzung hierfür ist die absolute, während des Laserschneidens unveränderliche Planlage des Folienwerkstüicks im Hinblick auf die eingestellte Fokusebene. Wird das Folienmaterial - aus welchen Gründen auch immer - aus der festgelegten Fokusebene herausbewegt und ist die Tiefenschärfe des Laserstrahls verfahrensbedingt gering, so wird in der Regel das Laserschneiden zwar nach wie vor stattfinden, die Schnittspaltbreite jedoch ist dann erheblich größer als berechnet. Daraus resultiert ein wesentlich größerer effektiver Laserstrahl-Durchmesser - ganz gleich ob die Bewegung nach unten oder nach oben von der Fokusebene erfolgt - und demzufolge eine messbare Vergrößerung aller Konturabmessungen, was das Einhalten der Maßtoleranzen erschwert oder gar unmöglich macht [7].

\section{Aspekte des qualitätsoptimierten Laser- schneidens metallischer Präzisionsfolien}

Unter sonst konstanten Laserschneidbedingungen und bei gleichem Werkstoff kommt der zu erzeugenden Konturform eine erhebliche Bedeutung im Hinblick auf die angestrebte hohe Fertigungsqualität zu. Dies kann unter Umständen dazu führen, dass bei komplizierten Konturen mit häufigen Ecken, Wendungen oder Schlitzen keinesfalls die wirtschaftlich optimale sowie verfahrenstechnisch zulässige Schneidgeschwindigkeit angewendet werden kann, da dann die Qualitätsanforderungen nicht erfüllt werden können.

In solchen Fällen besteht die Notwendigkeit, die Schneidgeschwindigkeit zu reduzieren, was nicht nur die Wirtschaftlichkeit des Laserschneidens gravierend senkt, sondern auch zu Beeinträchtigungen der restlichen, für diesen Bearbeitungsfall bereits optimierten Prozessparameter fuihren und unter Umständen sogar die Fertigungsqualität aus anderen Gründen negativ beeinflussen kann $[1,6]$.

Unten sonst konstanten Schneidbedingungen bei gleichem Werkstoff und gleichbleibender Foliendicke beeinflussen zwei Größen die Schnittspaltbreite gravierend: zum einen die Pulsenergie (davon die Komponente „Pulsbreite“ am stärksten), zum anderen die Fokuslage in Bezug auf die Werkstïckoberfläche. Je nach den ge- 
wählten Laserschneidbedingungen und der Dicke des Folienmaterials entsteht eine Schnittfuge, die in der Regel auf der Laserstrahl-Eintrittsseite erheblich breiter ist als auf der Laserstrahl-Austrittsseite der Metallfolie. Diese Schnittspalteigenschaft bezeichnet man als Konizität.

Die Schnittspaltbreite und die Konizität entscheiden letztlich über die Einhaltung der geforderten Maßtoleranzen. Es ist einleuchtend, dass beim Ablauf eines bestimmten $\mathrm{CNC}$-Programms eine vorausberechnete Laserstrahlführung erfolgt. Je nach Schnittspaltbreite und Konizität jedoch könnte an jede Stelle des gelaserten Werkstiicks eine korrekte Abmessung, Untermaß oder aber auch Übermaß entstehen. Eine derartige Unbestimmtheit ist absolut untolerierbar, da sie die wichtigste Qualitätsanforderung - das Einhalten der vorgegebenen Maßtoleranzen - buchstäblich ignoriert bzw. zunichte macht $[1,6]$.

Bei der CNC-Programmierung geschlossener Konturen bestehen zwei Möglichkeiten zur Berüicksichtigung der Schnittspaltbreite - entweder lässt man eine softwaremäßig erfolgende, automatische Schnittspaltkompensation durch äquidistante Verrechnung der Konturen zu oder man macht diese Verrechnung selbst und ändert alle Werkstïckmaße unter Berüicksichtigung der tatsächlichen Schnittspaltbreite. Bei offenen Konturen ist nur der zweite, uiberaus muihsame Weg der Verrechnung möglich. In allen Fällen jedoch werden die im Prozess auftretenden Werte für Schnittspaltbreite und Konizität sowie der Nachweis, dass sie während des Laserschneidens annähernd konstant bleiben, im Voraus noch bevor mit der lasertechnologischen Abwicklung der gestellten Aufgaben begonnen wird - benötigt.

Bedauerlicherweise ist es bis heute nicht möglich, anhand von Modellen brauchbare Ergebnisse für die Vorausberechnung der Schnittspaltbreite und der Konizität beim Laserschneiden dünner Metallfolien zu erhalten, obwohl der Bedarf an in der Praxis einsetzbaren Vorausberechnungsmethoden derzeit sehr groß ist $[1,6]$.

Es verbleibt nur die Möglichkeit, experimentell die benötigten Werte im Voraus zu bestimmen. Hierfür ist der einfache Strichversuch, bei welchem mehrere geradlinige Schlitze (offene Konturen) unter Änderung der gewünschten Parameter von Strich zu Strich in einer Probe gelasert werden, gut geeignet. Seine Vorteile sind darin zu sehen, dass er einfach durchfuihrbar ist und relativ schnell Orientierungswerte liefert. Andererseits sind die Nachteile, in erster Linie lasertechnologischer Natur, erheblich.

Im LSAZ-LaserLabor entstand der Entwurf eines handlichen (25 x $\left.40 \mathrm{~mm}^{2}\right)$, leicht herstellbaren, multivalent verwendbaren Testplättchens zur experimentellen Vorausbestimmung unter anderem der Schnittspaltbreite und der Konizität (Abbildung 4).

Darüber hinaus lassen sich anhand dieses Testplättchens Beobachtungen und Messungen der Gratbildung, der eventuellen Oberflächendefekte und der Schnittflächen

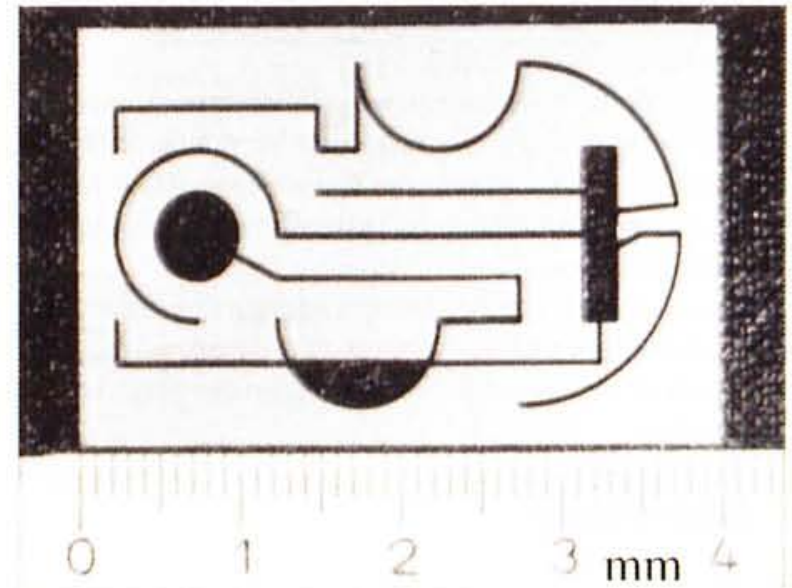

Abb. 4: Testplättchen des LSAZ-LaserLabors zur Bestimmung der Schnittspaltbreite, korrosionsbeständiger, austenitischer Stahl X10CrNi18-8 mit einer Materialstärke von $300 \mu \mathrm{m}$; Pulsfrequenz $280 \mathrm{~Hz}$; Spannung 200 $\checkmark$; Pulsdauer $185 \mu \mathrm{s}$; Fokusabstand 1,0 mm; Fokuslage - 12 mm; Prozessgas Stickstoff; Druck 16 bar; Düsendurchmesser 1,0 mm; Schneidgeschwindigkeit 190 mm/min; Trennmittel Hebrosynt 20; mittlere Schnittspaltbreite der Strahleintrittsseite $150 \mu \mathrm{m}$ und der Strahlaustrittsseite $134 \mu \mathrm{m}$;

des Laserstrahlgangs durchfuihren. Abweichungen von der Rundheit einer Bohrung lassen sich - falls relevant - genauso messen und beobachten wie das Verhalten mehrlagiger Folienpakete oder von Schutzfolien. Gegebenenfalls lassen sich Genaumaß, Untermaß oder Übermaß durch Vermessung zahlenmäßig genau ermitteln.

Die Testplättchen sind gut geeignet zum Aufbewahren und Archivieren bzw. als materieller Datenträger beim Aufbau von Datenbanken. Ferner können vergleichende Untersuchungen an den Testplättchen zweier Laseranlagen leicht durchgeführt werden. Darüber hinaus können derartige Testplättchen beim Abschluss einer Aufgabe als Qualitätsmuster dem Kunden übergeben werden. Beim Einreichen von Angeboten können die verschiedenen Qualitäten bei unterschiedlichen Schneidbedingungen verdeutlicht werden, d. h. dem Kunden wird die Auswahl einer bestimmten Qualitätsstufe erleichtert. Nicht zuletzt sind diese Testplättchen hervorragend für weitergehende Vermessungen oder metallkundliche Untersuchungen geeignet $[1,6]$.

\section{Laserschneiden von mehrlagigen Folienpaketen}

Die mehrlagige Bearbeitung flacher Werkstücke ist bei anderen 2D-Fertigungsverfahren, z. B. beim Stanzen, Elektroerodieren oder Wasserstrahlschneiden, nicht neu. Beim Laserschneiden dünner Metallpräzisionsfolien wurde dies bislang nicht praktiziert. Bei dieser Technologie werden anstelle der einlagigen Werkstiicke mehrlagige Folienpakete gleichzeitig aufgespannt und gelasert. Die wesentlichen Vorteile des Verfahrens „Laserschneiden von mehrlagigen Folienpaketen" $[1,3,6]$ sind:

- die Herstellung gratarmer Werkstuicke höchster Oberflächenqualität ist möglich,

- das Laserschneiden flacher Folienwerkstiucke aus gleichem Werkstoff oder aus unterschiedlichen Materialien sowie aus Folien gleicher bzw. unterschiedlicher 
Materialstärke unter Einhaltung auch engster Maßtoleranzen ist möglich,

- die gezielte Anwendung von Hilfsfolien ist möglich, z. B. zum Stïtzen von Folien im Stärkenbereich von $5 \mu \mathrm{m}$ bis $50 \mu \mathrm{m}$ gegen die Gaswirkung (Haltefolien) und/oder zum Erzielen höchster Laserschneidqualität (Schutzfolien),

- eine mehrfache Produktivitätssteigerung durch Verkürzung der Laserschneidzeiten ist erreichbar; dadurch ist eine erhebliche Senkungen der Stüickkosten möglich,

- die Aufspann- und Folienwechselzeiten werden signifikant reduziert.

In Abbildung 5 ist die Kantenqualität beim Schneiden eines dreilagigen Folienpaketes aus dem CrNi-Stahl X10CrNi18-8 dargestellt. Die Einzeldicke jeder Folie beträgt $50 \mu \mathrm{m}$. In den rasterelektronenmikroskopischen Aufnahmen ist die Schnittkantenqualität deutlich erkennbar. Die obere Folie (linkes Bild, Abb. 5) ist von hervorragender Qualität und nahezu gratfrei. Auch die mittlere Folie (rechtes Bild, Abb. 5) weist eine hohe Kantenqualität auf. Die dritte Folie (hier nicht abgebildet) wurde als Schutzfolie zur Schneidqualitätsverbesserung benutzt $[1,6]$.

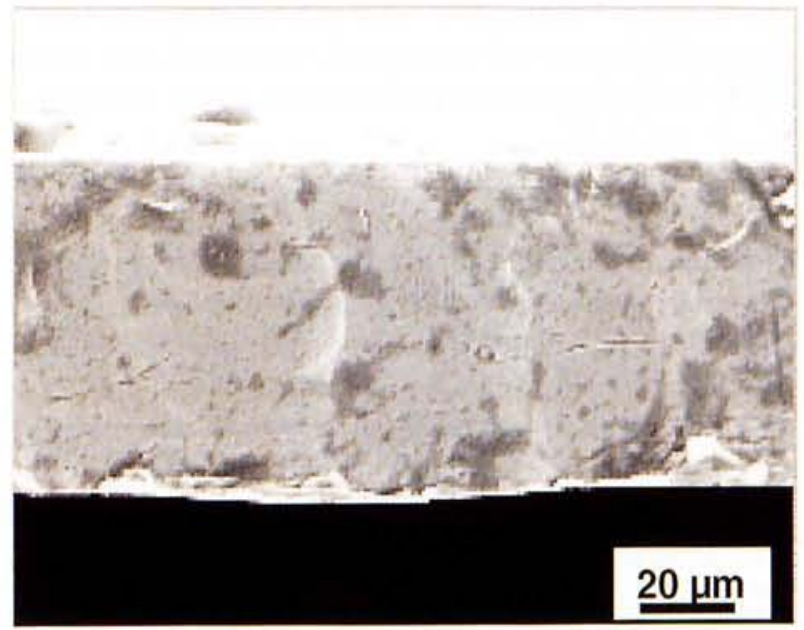

Abb. 5: Rasterelektronenmikroskopische Aufnahmen des Querschnittes durch ein Folienpaket aus drei Folien (links - obere Folie, rechts - mittlere Folie):

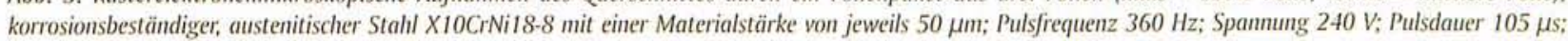
Fokusabstand $0.8 \mathrm{~mm}$; Fokuslage -20 $\mu \mathrm{m}$; Prozessgas Stickstoff; Druck 20 bar; Düsendurchmesser $0.5 \mathrm{~mm}$; Schneidgeschwindigkeit $150 \mathrm{~mm} / \mathrm{min}$; Trenmmittel Hebrosynt 20.

Das Wesentliche am neuartigen Bearbeitungsverfahren „Laserschneiden von mehrlagigen Folienpaketen“ ist die unabdingbare Notwendigkeit, geeignete Trennmittel zu verwenden, um die Trennung der einzelnen Folienlagen zu erleichtern bzw. überhaupt zu ermöglichen, ferner um die angestrebte hohe Oberflächenqualität beim Laserschneiden zu erreichen und die wirtschaftlichen Vorteile der Produktivitätssteigerung nutzen zu können $[1,6]$.

\section{Trennmitteleinsatz beim Laserschneiden von Metallpräzisionsfolien}

Beim Gießen in Metallformen (Kokillen- bzw. Druckgießen) sorgen geeignete Trennmittel dafuir, dass das flüssige Metall während und nach der Erstarrung nicht

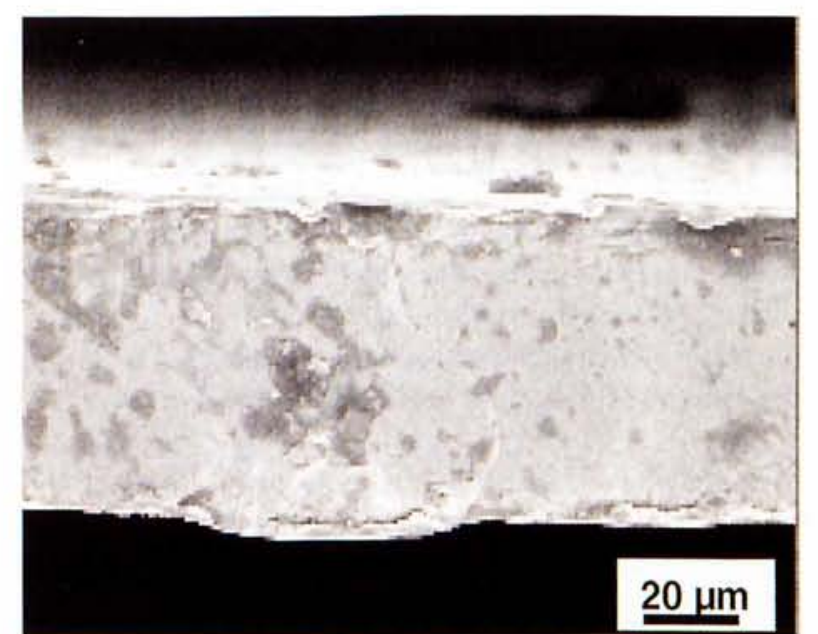

mit dem Formwerkstoff verschweißt, dort anhaftet oder anderweitig reagiert. Auch bei Schweißarbeiten werden Spezialtrennmittel eingesetzt, die verhindern sollen, dass die entstehenden Spritzer sowie die Schmelzperlen nicht auf Dauer auf den Funktionsoberflächen der Werkstücke anhaften können bzw. sich von dort mühelos entfernen lassen.

In manchen Laserbearbeitungsbetrieben wird das leidige Qualitätsproblem der vielfältigen Oberflächenschäden beim Laserschneiden (Spritzer, Schmelzperlen, Schmelzbärte, Grat- und Staubpartikel) mit Hilfe von konventionellen Trennmitteln bekämpft. In manchen Bereichen des Laserschneidens haben sich Trennmittel aus der Gießereitechnik gut bewährt, allerdings werden sie dann eher sporadisch und nur fuir bestimmte Aufgaben angewandt.

Die Ergebnisse der im LSAZ-LaserLabor durchgeführten, umfassenden Untersuchungen zur Anwendung des für das Laserschneiden entwickelten, wasserlöslichen Trennmittels Hebrosynt 20 [9] sind überaus positiv, sowohl bei einlagigem Lasern von Werkstïcken als auch bei der 2DLaserbearbeitung von mehrlagigen Folienpaketen $[1,6,8]$. 
bekannt ist, dass die Laserstrahl-Eintrittsseite der Werkstiicke nicht oder sehr wenig durch das Laserschneiden beeinträchtigt wird und dass hauptsächlich die Qualität der Laserstrahl-Austrittsseite der Folien durch das Lasern erheblich gemindert wird.

Die Folien, die zum Einsatz beim Laserschneiden von mehrlagigen Folienpaketen kamen, wurden stets einzeln und beiderseitig mit dem Trennmittel besprüht; anschließend wurden sie zu einem Paket zusammengelegt.

Bei den Untersuchungen zeigte sich, dass die qualitätssteigernde Wirkung des Trennmittels bei frisch besprühten, während des Laserschneidens noch nassen Werkstuickoberflächen erheblich stärker ist, als im Falle, dass die Trennmittel-Schicht vor Beginn des Laserschneidens ausgetrocknet ist. Dies ist ein zusätzliches Argument für die Anwendung von stationären Überflut-Systemen zum vollständigen Überfluten der Werkstuicke samt Aufspann-Vorrichtung mit einer Trennmittel-Lösung $[1,6,8]$. Im LSAZ-LaserLabor wurde neben dem Verfahren zum Anbringen des Trennmittels durch Besprühen auch ein Versuchsaufbau mit dem Ziel untersucht, die Eignung von in der Laserbearbeitungsanlage integrierten Überflut-Systemen für flüssige Trennmittel-Lösungen zur Qualitätsverbesserung beim Laserschneiden ein- oder mehrlagiger Folien-Werkstüicke zu überprüfen und eine optimale Zusammensetzung des Arbeitsmediums zu ermitteln.

Im Arbeitsraum der Laserschneidanlage wurde eine Wanne so angebracht, dass sie und der gesamte Aufbau der Überflut-Vorrichtung den Laserschneidprozess in keiner Weise beeinträchtigen. Im Innenraum der Wanne wurde eine Aufspann-Vorrichtung samt der zu lasernden Folien-Werkstücke montiert. Die Wanne wurde mit einem flüssigen Arbeitsmedium aufgefüllt. Der Flüssigkeitsspiegel lag 3 bis $5 \mathrm{~mm}$ über der laserstrahlzugewandten Werkstüickoberfläche $[1,6,8]$.

Nach mehreren Versuchen fand man eine Arbeitsmedium-Zusammensetzung (Verhältnis a) zu b) zu c) wie 1:2:3), die die gestellten Anforderungen in jeder Hinsicht erfuillt:

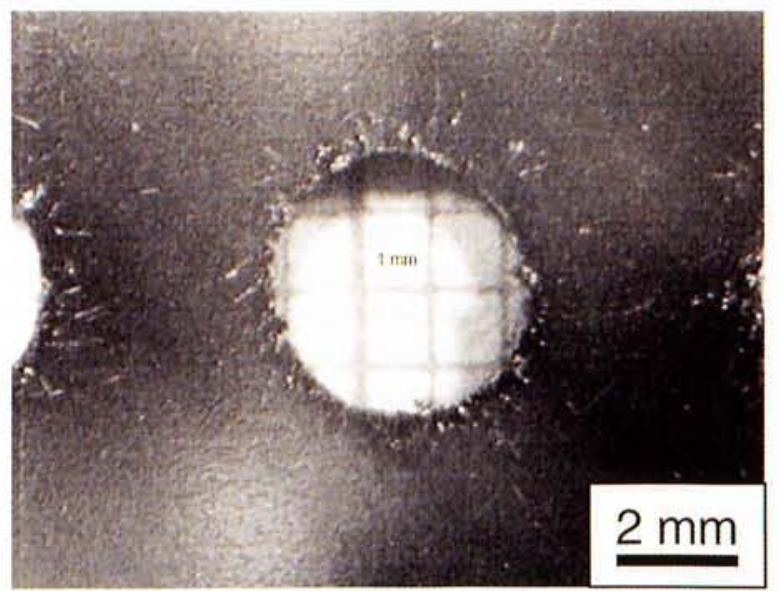

a) Wasser,

b) Glyzerin, eine Substanz, die unter anderem die folgenden Merkmale aufweist:

- hervorragender Korrosionsschutzmittel für die Bauteile und Werkstiicke,

- verhindert bzw. verlangsamt den Wasser-Dissoziationsprozess in $\mathrm{H}^{2}$ und $\mathrm{O}^{2}$,

- hat antibakterielle Wirkung und verlangsamt das Altern der Trennmittel-Lösung,

- begünstigt das Sedimentieren und das Herausfiltern der Schneidprodukte,

- ist wasserlöslich und hat ähnliche optische Eigenschaften wie Wasser sowie

c) Trennmittel, in diesem Fall Hebrosynt 20 [9].

Zu Beginn des Schneidvorgangs wird die Gaszufuhr eingeschaltet, erst danach kann der Laserstrahl die Werkstiickoberfläche erreichen. Das koaxial und unter Druck aus der Düse herausströmende Prozessgas verdrängt das flüssige Arbeitsmedium nach allen Seiten - je nach Gasdruck in einem kreisförmigen Bereich von einigen Millimetern bis wenigen Zentimetern Durchmesser $[1,6,8]$.

Unter diesen Bedingungen findet der Schneidvorgang so statt, als gäbe es kein flüssiges Arbeitsmedium. Die Spitze der Schneiddiuse kommt zu keinem Zeitpunkt in Berührung mit dem flüssigen Arbeitsmedium; sie wird während des Schneidens nicht mal nass. Wegen des ausströmenden Prozessgases ist es genauso unmöglich, dass Arbeitsmedium-Tröpfchen im Strahlführungssystem oder bis zur Fokussierungslinse vordringen $[1,6,8]$.

In Abbildung 6 sind die Vorteile des Überflut-Verfahrens hinsichtlich Schneidqualität, Maßhaltigkeit und Oberflächengüte verdeutlicht. Es galt, mehrere Bohrungen mit einem Durchmesser von $3,56 \mathrm{~mm}$ in Folienwerkstiicken aus dem unlegierten Stahl St 37 mit einer Materialstärke von $100 \mu \mathrm{m}$ durch Laserschneiden herzustellen. Die Werkstïcke wurden stets zweilagig, d. h. im Paket zusammen mit einer Schutzfolie gelasert. In Abbildung 6 ist jeweils die Unterseite der obenliegenden Werkstückfolien dargestellt, links aus den Vergleichsversuchen im Trockenschnitt, rechts aus den Überflut-Untersuchungen mit Trennmitteln.

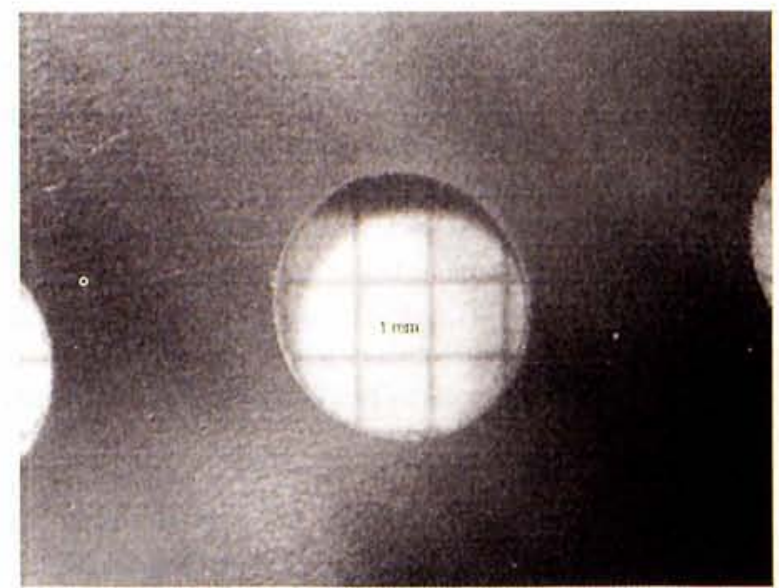

Abb. 6: Laserbohrungen von 3,56 mm Durchmesser, Material unlegierter Stahl St 37; Materialstärke 100 Hm, zweilagige Paketbearbeitung mit Schutzfolie; dargestellt ist jeweils die Unterseite der obenliegenden Werkstïckfolien (links ohne Trennmittel, rechts mit dem Trennmittel Hebrosynt 20); Pulsfrequenz 360 Hz; Spannung 240 V; Pulsdauer $105 \mu$ s; Fokusabstand 1,0 mm; Fokuslage -25 $\mu \mathrm{m}$; Prozessgas Stickstoff: Druck 18 bar; Düsendurchmesser 0,5 mm; Schneidgeschwindigkeit 180 mm/min. 
Beim Laserschneiden offener Konturen (Abb. 7) ist die Entstehung lokaler, verfahrensbedingter, meist punktueller Einstich-Oberflächenschäden unvermeidlich. In solchen Fällen gilt, die Kraterbildung und die Oberflächenschäden zu minimieren, was sinnvollerweise in erster Linie durch das Variieren der Schneidparameter erfolgt. Darüber hinaus lassen sich durch den Einsatz von Trennmitteln weitere Erfolge bei der Schädenminimierung erzielen.

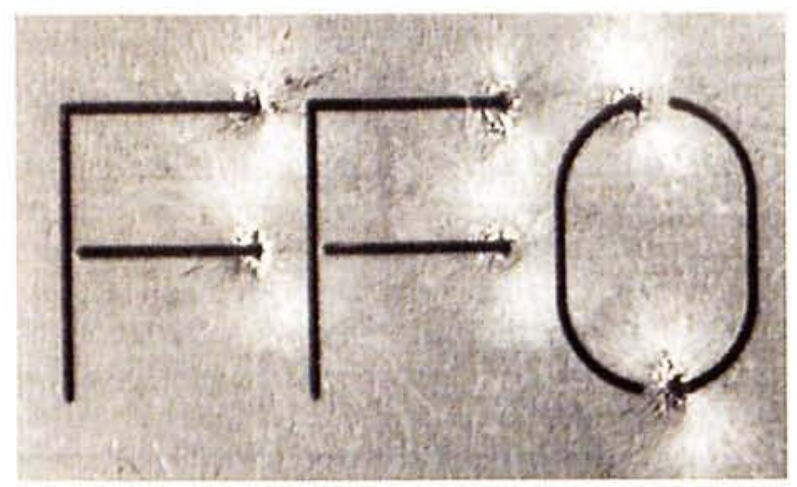

Abb. 7: Laserschneiden offener Konturen - Minimierung von Einstichpunkt Oberflächenschäden; Material unlegierter Stahl St 37; Materialstärke 300 um, Pulsfrequenz $120 \mathrm{~Hz}$; Spannung 255 V; Pulsdauer $220 \mu$ s; Fokusabstand $0,8 \mathrm{~mm}$; Fokuslage - $15 \mu \mathrm{m}$; Prozessgas Sauerstoff; Druck 9 bar: Düsendurchmesser 1,0 mm; Schneidgeschwindigkeit $100 \mathrm{~mm} / \mathrm{min}$; Trennmittel Hebrosynt 20; Schrifthöhe 4,0 mm; Schnittspaltbreite $130 \mu \mathrm{m}$.

\section{Laserschneiden von Präzisionsfolien aus Hartstoff-Metall-Verbunden}

Hartstoff-Metall-Verbunde (HMV) stellen eine neue, vielversprechende Entwicklung bei den in der Fertigungstechnik anwendbaren Hartmaterialien dar. Die Härte und die Sprödigkeit der synthetischen Diamanten bzw. der Partikel aus kubischem Bornitrid (CBN) wird mit den Eigenschaften einer flexiblen Metallmatrix kombiniert. Damit will man vor allem eine ausreichende Duktilität des Verbundmaterials bei einer hohen Härte erreichen [8].

Die Phosphorbronze CuSn6, eine Kupferbasis-Knetlegierung, bildet in der Regel die Metallmatrix der HMVFolien. Aus den bisherigen Untersuchungen ist bekannt, dass sich Kupferwerkstoffe, bedingt durch die geringe Absorption und die große Wärmeleitfähigkeit des Kupfers, weitaus schwerer durch Laserschneiden bearbeiten lassen, als die gebräuchlichen Stahlqualitäten. Daher ist in diesem Fall eine genaue Fokussierung des Laserstrahls auf der Werkstïckoberfläche unter bereits vorher optimierten Schneidbedingungen von entscheidender Bedeutung für das Gelingen der Laserschneidbearbeitung und die Erfüillung der gestellten Qualitätsanforderungen. Zudem besitzt die CuSn6-Folie eine geringe Steifigkeit, was zum „Ausweichen“ unter dem Gasstrom führt. Dadurch kommt es zu abrupten Unterbrechungen des Laserschneidprozesses [8].

Das Laserschneiden von Folienwerkstücken aus Diamant-Metall-Verbunden (DMV) darf keinesfalls zur Gratbildung führen. Darüber hinaus sollten die Diamantkristalle durch den Laserschneidprozess nicht aus der Metallmatrix gelöst werden. Ansonsten verschlechtern sich Standzeit und Arbeitsgenauigkeit der fertiggelaserten Trennscheiben erheblich.

Durch 2D-Laserschneiden einlagiger Werkstïcke aus HMV-Folien im Trockenschnitt und ohne SchutzfolienUnterlagen können erfolgreich Trennscheiben hergestellt werden. Nach einer Laserparameteroptimierung werden die gestellten hohen Anforderungen hinsichtlich Schneidqualität und Einhaltung enger Maßtoleranzen in jeder Hinsicht erfüllt [8].

Eine besondere Aufmerksamkeit wurde den Maßnahmen zur Verminderung bzw. zur völligen Unterdrückung der Schwärzung entlang der Schnittspaltränder der Strahlaustrittsseite der Werkstiicke aus DMV-Folien gewidmet. Diese Schwärzung wurde ausschließlich beim Laserschneiden von diamanthaltigen HMV-Folien beobachtet.

Es ist naheliegend, dass die beobachtete Schwärzung eine Folge der Reaktionen ist, die zwischen den Diamantkörnern, der verfluissigten Metallphase und dem Luft-Sauerstoff bei den herrschenden hohen Temperaturen stattfinden. Bei der dann ablaufenden Verbrennung bzw. Teiloxidation der Diamantkörner entstehen offensichtlich Rußpartikel, die in allen Richtungen verwirbelt werden und sich bevorzugt entlang der kühleren Schnittspaltränder der Strahlaustrittsseite der DMV-Werkstiukke als Schwärzung ausscheiden.

Die aussichtsreichste Variante zur völligen Unterdrükkung dieser unerwünschten Schwärzung durch Unterbinden der Zufuhr an Luft-Sauerstoff ist die Anwendung von stationären Systemen zum Überfluten der FolienWerkstuicke samt Aufspann-Vorrichtung mit einer flüssigen Arbeitsmedium-Lösung [8].

Zur Verbesserung der Schneidqualität können Schutzfolien aus der Knetlegierung CuSn6 mit einer Materialstärke von 100 bis $200 \mu \mathrm{m}$ verwendet werden. Allerdings sollte dies sinnvollerweise gleichzeitig mit der Anwendung von stationären Überflut-Systemen zum Überfluten der Folien-Werkstuicke samt Aufspann-Vorrichtung mit einer Trennmittel-Lösung erfolgen [8], um stets die problemlose wie auch beschädigungsfreie Entfernung der eingesetzten Schutzfolien zu gewährleisten.

\section{Zusammenfassung}

Metallpräzisionsfolien können durch Laserschneiden bearbeitet werden, wenn geeignete Halterungstechniken verwendet und die Verfahrensparameter optimiert werden. Konturfehler und Welligkeiten der Funktionsoberflächen werden dadurch weitestgehend vermieden. Vorteile des Laserschneidens sind die hohe Schneidqualität und die geringe Schnittspaltbreite. Das Laserschneiden von Folienpaketen stellt eine neuartige Technologie zur Bearbeitung von Metallpräzisionsfolien dar. Die Schnittkantenqualität variiert zwischen hervorragend und sehr gut. Der Einsatz geeigneter Trennmittel ist notwendig, um die Einzelfolien fehlerfrei zu trennen und die geforderte hohe Schneidqualität zu gewährleisten. 


\section{Danksagung}

Wir danken Herrn Dr. Banisch (T.IN.A. Brandenburg) und Herrn DK R. Ries (TFH Wildau) für die umfassenden Diskussionsbeiträge sowie die Unterstützung bei der Lösung von Teilaufgaben. Frau K. Kubica (TFH Wildau) sei für die Probenpräparation und die lichtmikroskopischen Untersuchungen gedankt. Dank gilt auch der Vollstädt Diamant GmbH für die Bereitstellung von hartstoffhaltigen Werkstïickfolien.

Die Arbeiten werden durch das BMBF, AiF-Forschungsprojekt FKZ 1702199, gefördert.

\section{Literatur}

[1] Richter, A.; Erinski, D.: „Laserschneiden von Metallpräzisionsfolien“, Vortrag, 14. Int. Wiss. Konferenz Mittweida IWKM 2000, Tagungsgruppe „Lasertechnik“, in „Scientific Reports", wiss. Zeitschrift der Hochschule Mittweida (FH), Band K - Lasertechnik, S. 33-40, Mittweida, 08.11.2000

[2] Heikenwälder, J.: „Wirkungsweise und Einsatzgebiete des wasserstrahlgeführten Laserschneidens in der Präzisionsbearbeitung", Vortrag, 14. Int. Wiss. Konferenz Mittweida IWKM 2000, Tagungsgruppe „Lasertechnik“, in „Scientific Reports", wiss. Zeitschrift der Hochschule Mittweida (FH), Band K - Lasertechnik, S. 73-80, Mittweida, 09.11.2000

[3] Erinski, D.: Laserschneiden von Metallpräzisionsfolien, VDI-Nachrichten Nr. 38, Sonderteil „Berlin-Brandenburg“, Nr. 4/2000, VDI Verlag, Duisseldorf, 22.09.2000

[4] Roth, S.; Geiger, M.: Excimerlaser-Bearbeitung: klare Strukturen unter dem Flüssigkeitsfilm, Mittelung aus dem Lehrstuhl für Fertigungstechnologie, Friedrich-AlexanderUniversität, Erlangen-Nürnberg, 30.08.2000

[5] Heikenwälder, J. u. a.: „Wasserstrahlgefuihrte Laser in der Präzisionsbearbeitung“, Laserpraxis, 15 (2000) 6, S. 36-40

[6] Richter, A.; Erinski, D.: Zwischenbericht über die Durchführung und den Stand des Vorhabens „Laserschneiden von Metallpräzisionsfolien“, AiF „Otto von Guericke“, FKZ 1702199, TFH Wildau und LSAZ-LaserLabor, 28.04.2000

17] Erinski, D.: Laserschneiden von ReinWolfram-Folien, Ergebnisbericht, LSAZ-LaserLabor, Frankfurt (Oder), 19.02.2001

181 Erinski, D.: Laserschneiden von Hartstoff-Metall-Verbunden (HMV), Ergebnisbericht, LSAZ-LaserLabor, Frankfurt (Oder), 08.08.2000

[9] Produkt-Information: Spezial-Trennmittel zum Laserschneiden Hebrosynt 20, Artikel-Nr. F080, Hebro Chemie $\mathrm{GmbH}$ in Mönchengladbach, Merkblatt, Juli 2000

[10] Petring, D.; Schneider, F; Thelen, C.; Poprawe, R.: Neue Entwicklungen zum Laserschneiden von Fein- und Feinstblechen, LaserOpto, 31 (1999) 2, S. 70

[11] Lerner, E. J.: Industrial laser cutting goes mainstream, Laser Focus World, 35 (1999), pp. 113-114, 116-118

[12] Petring, D.: Neue Entwicklungen im Bereich des Laserstrahlschneidens, Tagungsband AKL. 1998, Aachener Kolloquium Lasertechnik 1998, Verlagsgesellschaft Grütter, Hannover, 1998

[13] DIN 2310, Teil 6: Thermisches Schneiden, Gruppe 3.4.1.5 Thermisches Abtragen durch Laserstrahl, Untergruppen 3.4.1.5.3 Laser-Brennschneiden, 3.4.1.5.4 Laser-Schmelzschneiden und 3.4.1.5.5 Laser-Sublimierschneiden, Beuth Verlag, Berlin und Köln, Ausgabe Oktober 1995

\section{Autoren}

Prof. Dr. Asta Richter

Technische Fachhochschule Wildau Fachrichtung Physikalische Technik Werkstoff-, Laser- und Oberflächentechnik Tel. (0 33 75) 508-219

E-Mail: richter@pt.tfh-wildau.de

\section{Dr. Dobri Erinski}

Laser- und Strahltechnik ApplikationsZentrum Ostbrandenburg (LSAZ)

LaserLabor

Im Technologiepark 1

15236 Frankfurt (Oder)

Tel. (03 35) 6802603

E-Mail: LaserLabor.Erinski@t-online.de 